\title{
MATHEMATICAL NOTE
}

\section{IRROTATIONAL FLOW OF A LIQUID, WITH AXIAL SYMMETRY}

\author{
by D. H. PARSONS
}

(Received 12th November, 1962)

1. In the case of two-dimensional liquid motion, knowing one irrotational flow, we can at once deduce any number of others by conformal transformations. But in the case of axially symmetric motion, there is no corresponding device. Accordingly, it is useful to examine other methods, by which one axially symmetric motion can be deduced from another.

First of all, any change of variables on the co-ordinates and the Stokes' stream function, or on the co-ordinates and the velocity potential, which leaves unchanged the appropriate partial differential equation, provides such a transformation from one flow to another. I have recently shown (1) that these transformations are of three kinds only, namely the following: (i) trivial transformations, consisting of multiplications of the co-ordinates by a constant scale factor, change of origin on the axis of symmetry, reversal of the positive direction of the axis of symmetry, multiplication of the velocity potential or of the Stokes' stream function by a constant, addition to the velocity potential of a particular integral of the equation satisfied by the velocity potential, addition to the Stokes' stream function of a particular integral of the equation satisfied by Stokes' stream function; (ii) Kelvin's transformation, that is, if $\phi$ be the velocity potential, and $r, \theta$ be spherical polar co-ordinates, then the transformation

$$
R r=k^{2}, \quad \Theta=\theta, \quad \Phi=(A / k) r \phi,
$$

where $A, k$ are non-zero constants, leads to a new velocity potential $\Phi$ in terms of the spherical polar co-ordinates $R, \Theta$; (iii) if $\psi$ be the Stokes' stream function of an irrotational flow, the transformation

$$
R r=k^{2}, \quad \Theta=\theta, \quad \Psi=-B k \psi / r,
$$

$B$ and $k$ being non-zero constants, leads to a new Stokes' stream function $\Psi$, in terms of $R, \Theta$. This transformation, like Kelvin's, involves geometrical inversion with respect to a point on the axis.

2. We now examine a device of a different character. Let $\phi$ and $\psi$ be the velocity potential and Stokes' stream function respectively, of an axially symmetric motion which we suppose known. Let us use cylindrical polar 
co-ordinates $z, \omega$, so that the equations governing the motion are

$$
\left.\begin{array}{r}
\frac{\partial^{2} \phi}{\partial z^{2}}+\frac{\partial^{2} \phi}{\partial \omega^{2}}+\frac{1}{\omega} \frac{\partial \phi}{\partial \omega}=0 \\
\frac{\partial^{2} \psi}{\partial z^{2}}+\frac{\partial^{2} \psi}{\partial \omega^{2}}-\frac{1}{\omega} \frac{\partial \psi}{\partial \omega}=0 \\
\frac{\partial \psi}{\partial z}+\omega \frac{\partial \phi}{\partial \omega}=0 \\
\frac{\partial \psi}{\partial \omega}-\omega \frac{\partial \phi}{\partial z}=0
\end{array}\right\}
$$

the first two being consequences of the last two. Consider the function

$$
\xi=(z \phi-\psi) / l,
$$

$l$ being a non-zero constant, having the dimensions of a length. Then we have, identically,

$\frac{\partial^{2} \xi}{\partial z^{2}}+\frac{\partial^{2} \xi}{\partial \omega^{2}}+\frac{1}{\omega} \frac{\partial \xi}{\partial \omega}$

$$
\left.\begin{array}{rl}
\equiv \frac{1}{l}\left[z\left\{\frac{\partial^{2} \phi}{\partial z^{2}}+\frac{\partial^{2} \phi}{\partial \omega^{2}}+\frac{1}{\omega} \frac{\partial \phi}{\partial \omega}\right\}\right. & \left.-\left\{\frac{\partial^{2} \psi}{\partial z^{2}}+\frac{\partial^{2} \psi}{\partial \omega^{2}}-\frac{1}{\omega} \frac{\partial \psi}{\partial \omega}\right\}\right], \\
& -\frac{2}{\omega}\left\{\frac{\partial \psi}{\partial \omega}-\omega \frac{\partial \phi}{\partial z}\right\}
\end{array}\right]
$$

and therefore, by (1),

$$
\frac{\partial^{2} \xi}{\partial z^{2}}+\frac{\partial^{2} \xi}{\partial \omega^{2}}+\frac{1}{\omega} \frac{\partial \xi}{\partial \omega}=0
$$

Hence $\xi$, given by (2), is the velocity potential of a new axially symmetric motion.

Again, consider the function

$$
\eta=\left(\omega^{2} \phi+z \psi\right) / m,
$$

$m$ again being a non-zero constant, having the dimensions of a length. Then we have, identically

$$
\begin{aligned}
& \frac{\partial^{2} \eta}{\partial z^{2}}+\frac{\partial^{2} \eta}{\partial \omega^{2}}-\frac{1}{\omega} \frac{\partial \eta}{\partial \omega} \\
& \equiv \frac{1}{m}\left[\begin{array}{c}
\omega^{2}\left\{\frac{\partial^{2} \phi}{\partial z^{2}}+\frac{\partial^{2} \phi}{\partial \omega^{2}}+\frac{1 \partial \phi}{\omega \partial \omega}\right\}+z\left\{\frac{\partial^{2} \psi}{\partial z^{2}}+\frac{\partial^{2} \psi}{\partial \omega^{2}}-\frac{1 \partial \psi}{\omega \omega}\right\} \\
+2\left\{\frac{\partial \psi}{\partial z}+\omega \frac{\partial \phi}{\partial \omega}\right\}
\end{array}\right]
\end{aligned}
$$


and therefore, by (1),

$$
\frac{\partial^{2} \eta}{\partial z^{2}}+\frac{\partial^{2} \eta}{\partial \omega^{2}}-\frac{1}{\omega} \frac{\partial \eta}{\partial \omega}=0
$$

Hence $\eta$, given by (3), is the Stokes' stream function of a new axially symmetric, irrotational motion.

3. There is an interesting link between these transformations, (2) and (3), and the transformations (ii) and (iii) of $\S 1$. But before going further we observe the following curious feature of the functions $\xi$ and $\eta$.

From the velocity potential $\phi$, and Stokes' stream function $\psi$, of a known irrotational flow, we deduce two new irrotational flows, one whose velocity potential is $\xi$ as in (2), and another whose Stokes' stream function is $\eta$ as in (3). These flows are in general distinct. For it follows directly from (2) and (3) that, after taking account of (1),

$$
\begin{array}{r}
\frac{\partial \eta}{\partial z}+\omega \frac{\partial \xi}{\partial \omega} \equiv \frac{1}{\operatorname{lm}}\left\{(l-m) \omega\left(\omega \frac{\partial \phi}{\partial z}-z \frac{\partial \phi}{\partial \omega}\right)+l \psi\right\}, \\
\frac{\partial \eta}{\partial \omega}-\omega \frac{\partial \xi}{\partial z} \equiv \frac{\omega}{\operatorname{lm}}\left\{(l-m)\left(z \frac{\partial \phi}{\partial z}+\omega \frac{\partial \phi}{\partial \omega}\right)+(2 l-m) \phi\right\} .
\end{array}
$$

Now, if $l=m \neq 0$, it is evident that the right hand sides of (4) and (5) are never identically zero, so that $\xi$ and $\eta$ cannot correspond to the same flow. If $l \neq m$, it can be shown quite easily, changing to spherical polar co-ordinates (i.e., writing $z=r \cos \theta, \omega=r \sin \theta$ ), that the right hand sides of (4) and (5) can only be identically zero in the trivial case when

$$
\phi=r^{p} u(\mu), \quad \psi=\frac{1}{p+1} r^{p+1}\left(1-\mu^{2}\right) \frac{d u}{d \mu},
$$

where $p=(2 l-m) /(m-l), \mu=\cos \theta$, and $u$ satisfies Legendre's equation

$$
\left(1-\mu^{2}\right) d^{2} u / d \mu^{2}-2 \mu d u / d \mu+p(p+1) u=0 .
$$

Thus, except in the above trivial case, it is impossible to choose $l$ and $m$ so that the functions $\xi$ and $\eta$ correspond to the same flow.

Again, it is easily verified that, except in exactly the same trivial case mentioned above, the transformations (ii) and (iii), $\S 1$, applied to the functions $\phi(r, \theta)$ and $\psi(r, \theta)$ corresponding to a known flow, can never lead to functions $\Phi(R, \Theta)$ and $\Psi(R, \Theta)$ corresponding to the same flow, whatever the choice of the constants $A$ and $B$.

But we shall now show that if to a known flow, $f$ say (in the space of $r, \theta$ ), we apply, firstly the transformations (2) and (3), with $l=m=k$, and then, to the resulting (non-corresponding) velocity potential $\xi$ and Stokes' stream function $\eta$, apply respectively the transformations (ii) and (iii), §1, with $B=A$, we obtain 
two functions $\Phi$ and $\Psi$ which are indeed the corresponding velocity potential and Stokes' stream function of a new flow in the $R, \Theta$ space.

Writing $z=r \cos \theta, \omega=r \sin \theta, l=m=k$ in (2) and (3), and applying (ii) and (iii), $\S 1$, with $B=A$, to the resulting functions $\xi$ and $\eta$, we have

$$
\left.\begin{array}{rl}
\Phi & =\left(A / k^{2}\right)\left\{\left(r^{2} \cos \theta\right) \phi-r \psi\right\}, \\
\Psi & =-A\left\{\left(r \sin ^{2} \theta\right) \phi+(\cos \theta) \psi\right\}, \\
r & =k^{2} / R, \quad \theta=\Theta .
\end{array}\right\}
$$

Now, in spherical polar co-ordinates $r, \theta$, the equations corresponding to the last two equations (1) are

$$
\left.\begin{array}{r}
\partial \psi / \partial r+(\sin \theta) \partial \phi / \partial \theta=0 \\
\partial \psi / \partial \theta-\left(r^{2} \sin \theta\right) \partial \phi / \partial r=0 .
\end{array}\right\}
$$

But from (6), taking account of (7), we obtain directly

$$
\begin{aligned}
& \partial \Psi / \partial R+(\sin \Theta) \partial \Phi / \partial \Theta=0, \\
& \partial \Psi / \partial \Theta-\left(R^{2} \sin \Theta\right) \partial \Phi / \partial R=0,
\end{aligned}
$$

showing that $\Phi$ and $\Psi$ are respectively velocity potential and Stokes' stream function of a new flow, $F$ say, in the $R, \Theta$ space.

4. Further, we may remark that the relationship between the flows $f$ and $F$ above is symmetric. For if we apply to the functions $\Phi$ and $\Psi$ in (6), the transformations respectively corresponding to (2) and (3), still with $l=m=k$, namely (in spherical polar co-ordinates)

$$
\begin{aligned}
& \Xi=\{(R \cos \Theta) \Phi-\Psi\} / k, \\
& H=\left\{\left(R^{2} \sin ^{2} \Theta\right) \Phi+(R \cos \Theta) \Psi\right\} / k,
\end{aligned}
$$

we obtain (noticing that $R=k^{2} / r, \Theta=\theta$ ), after a little reduction,

$$
\begin{aligned}
& \Xi=(A / k) r \phi, \\
& H=-A k \psi / r .
\end{aligned}
$$

If, now, we apply respectively the transformations (ii) and (iii), $\S 1$, in reverse, still with $B=A$, i.e.

we have at once

$$
\phi^{*}=(k / A)(\Xi / r), \quad \psi^{*}=-(1 / A k) r H,
$$

$$
\phi^{*}=\phi, \quad \psi^{*}=\psi,
$$

so that we retreive the original flow $f$.

\section{REFERENCE}

(1) D. H. Parsons, J. Lond. Math. Soc., 34 (1959), 442-8.

\section{THE UNIVERSITY}

READING 